\title{
Compressing Information of Target Tracking in Wireless Sensor Networks
}

\author{
Jianzhong Li, Qianqian Ren \\ Department of Computer Science and Technology, Harbin Institute of Technology, Harbin, China \\ E-mail: \{lijzh, qqren\}@hit.edu.cn \\ Received January 21, 2011; February 15, 2011; February 22, 2011
}

\begin{abstract}
Target tracking is a well studied topic in wireless sensor networks. It is a procedure that nodes in the network collaborate in detecting targets and transmitting their information to the base-station continuously, which leads to data implosion and redundancy. To reduce traffic load of the network, a data compressing based target tracking protocol is proposed in this work. It first incorporates a clustering based data gather method to group sensor nodes into clusters. Then a novel threshold technique with bounded error is proposed to exploit the spatial correlation of sensed data and compress the data in the same cluster. Finally, the compact data presentations are transmitted to the base-station for targets localization. We evaluate our approach with a comprehensive set of simulations. It can be concluded that the proposed method yields excellent performance in energy savings and tracking quality.
\end{abstract}

Keywords: Wireless Sensor Networks, Target tracking, Compressing

\section{Introduction}

Target tracking is an important problem of wireless sensor networks (WSNs). It has been applied in various areas such as disaster predication, emergency response, battlefield surveillance, home and office control, etc. Many target tracking protocols have been proposed to support long-term surveillance by using large scale WSNs [1-5].

In the applications of target tracking with WSNs, the users are often interested in observing where the target is at each time interval and figuring its trajectory. In such cases, continuous information reporting of the target is required. In continuous surveillance, sensor nodes in the network collaborate in detecting the target, measuring the signal the target emitting and transmitting measurements to the base-station for further processing. However, the limits of WSNs including limited bandwidth, processing capabilities and energy supply challenge the research of target tracking.

To minimize the volume of information transmission, we can process the information of targets in a distributed way in the network and transmit localization results to the base-station. In-network localization is an effective idea to reduce the volume of transmitted data, but is rather infeasible for multiple targets tracking that need high complexity computation, such as Kalman filter,
Particle filter and Bayesian transforms in targets decomposition. The limited computation capacity of sensor nodes may not be sufficient to perform complex operations at nodes.

Considering the scenarios of target tracking, sensor nodes generate sensed data of targets by measuring the signal they emit. Most physical signals decay with distance, thus readings of the sensor nodes have similar pattern if their distances to a target are approximately same. In other words, sensed data for targets usually exhibits a large degree of redundancy. Approximation is an efficient mean of data reduction, in which sensed data with similar patterns is replaced by an approximate value, and only the approximate values are transmitted to the base-station. Approximation can reduce the amount of sensed data that need to be transmitted with allowable accuracy scarifying.

In this paper, we present a data compressing based target tracking protocol, which incorporates data approximation algorithm in the procedure of targets tracking. The characteristic of sensed data over sensor nodes surrounding interested targets is exploited, replaced as a series of approximate values. Compact descriptions of these readings are transmitted to the base-station, where targets location is implemented on the compact descriptions directly. Given an error bound, we try to compress 
readings for the same target maximally by grouping the original data and approximating them falls within the error bound around estimated values. The proposed approach can release the traffic load and reduce energy consumption for data transmission efficiently. However, this often comes at the price of loss in tracking quality, which is equivalent to a loss in data precision and localization accuracy. We analyze the error of tracking results and discuss the determinations of parameters in the paper. In addition, the proposed compressing provides a low overhead, which makes it practical for overhead sensitive applications with WSNs.

The contributions of this paper are as follows:

- We introduce a new approximation scheme that makes full use of correlations among data of multiple sensor nodes. It exploits the spatial correlation of targets information to implement data reduction.

- We incorporate the idea of data reduction into target tracking, which shrinks the volume of transmitted data efficiently with guarantee of tracking quality. It is an efficient solution for prolonged network lifetime.

- We explore the trade-off between energy savings and tracking quality. We aim to design a tracking system in an energy-efficient manner at the price of allowable accuracy sacrificing.

- We provide an extensive experiments and analysis of our framework using a simulated sensor network. Experimental results show that our approach achieves well performance in terms of tracking quality and energy conservation.

The remainder of this paper is organized as follows. Related work is shown in Section 2. We give the network model in Section 3. A data compressing based tracking protocol is detailed in Section 4. We analyze the performance of proposed technique in Section 5. Section 6 presents detailed simulations. Finally, we conclude this paper in Section 7.

\section{Related Work}

In recent years, many research works have been provided in the area of target tracking using WSNs. The important issues studied mainly include energy efficient tracking and accurate tracking.

The authors in [6-9] adopt binary sensor model to track targets. The output of each binary sensor is only one bit ( 0 or 1$)$. The binary sensor nodes based tracking can conserve the energy for data transmissions efficiently. However, this kind of nodes does not have the capacity of calculation, and any loss of packets may affect the tracking accuracy evidently. Some works address the problem of energy efficiency by reducing the number of nodes participating in working. In [10], a distributed tracking algorithm using dynamic conveying tree structure is presented, which optimizes the problem of target tracking by building a convoy tree sequence with high tree coverage and low energy consumption. The work of [11] proposes an information-driven tracking approach by deciding the collaboration of sensor nodes considering the constraints of information and resource consumption.

The authors in [2] propose a minimal contour-tracking model to minimize the number of nodes involved in tracking. It searches for the minimal tracking area based on the vehicular kinematics to minimize working nodes. In [12], the problem of tracking mobile nodes is addressed by measuring the Doppler shifts of the transmitted signal. Moreover, the extended Kalman filter is adopted to remove the effects of the measurement errors in sensor networks with uncertainty. The distributed algorithms for in-network tracking and range queries are proposed in [13], they use differential one-form in the application of target tracking to search for a given identifiable target with low time complexity. The proposed approaches are also flexible to network changes and node mobility.

The above tracking techniques focus on reducing transmission amounts or the number of nodes participating in work.

To further reduce energy consumption in the longterm surveillance, nodes scheduling is applied in moving target tracking systems $[4,14,15]$. A real-time target tracking system with WSNs is designed in [3,4], which adopts an energy management scheme to make sensor nodes rotate in active and sleep state to conserve energy of the network. Moreover, some scheduling and wake-up topics are analyzed. In [14], an optimal node sleep scheduling protocol for rare-event detection is proposed. A deterministically rotating sensory coverage with constrains of detection delay is developed. The authors of [15] study the problem of network deployment and design an efficient scheduling protocol. It wakes up and shuts down sensor nodes with certain spatial and temporal preciseness.

These existing techniques mainly focus on the tracking and searching of a single target, it is not adaptable to multiple targets tracking. The authors of [2] study fault tolerant tracking. The Gaussian mixture model is introduced to capture the characteristics of the target signal. In addition, a temporally adaptive variant of the approach is proposed to track dynamic multiple targets under changing environments, with noisy considering. While the focus of [2] is accurately tracking moving targets with noise considering. In this paper, we propose a real time target tracking protocol with energy savings and 
tracking quality guarantee. We seek to exploit the redundancy of tracking information, compress the sensed data, and transmit the compressed data to the based-station. Our method not only reduces data transmission to the base-station, but also implements localization in compressed data structure directly.

\section{Network Model}

This section describes the network model used in this paper. To simplify the presentation, we give the network model based on following assumptions.

- A monitored area is covered by a large number of homogeneous sensor nodes with redundant density. Each node gets its own location via GPS or a certain localization technique.

- All sensor nodes in the monitoring area have the same sensing range, denoted as $R$. The sensing area of each sensor node is a disk centered at the node with radius $R$.

- Nodes in the network are organized as an adaptive clustering hierarchy [16]. Under the clustering based routing protocol, sensed data is routed to the destination.

Figure 1 gives an example of the network model. There are one cluster head node and multiple member nodes in each cluster. When a member node detects the target, it transmits sensed data to its cluster head. The cluster head node processes packets from its member nodes to obtain a compact data structure, which is transmitted the base-station.

\section{Compressing Based Target Tracking Protocol}

In this section, we first illustrate the general framework of data compressing based target tracking protocol (DCTTP), then present its working procedure in details.

\subsection{General Framework of DCTTP}

Target tracking is a procedure that nodes in the network collaborating in detecting and locating the given targets. When targets show up in a local area, nodes surrounding them (targets are insider their sensing range) detect the targets via measuring the signal they emit, generate sensed data and send it to cluster head nodes. After receiving packets from member nodes, the cluster heads suppress these data maximally with guarantee of tracking quality, and then transmit a compact data description to the based station for further processing to locate targets. DCTTP can be divided into four phases: 1) data collection, 2) data compressing, 3) data transmission and 4)

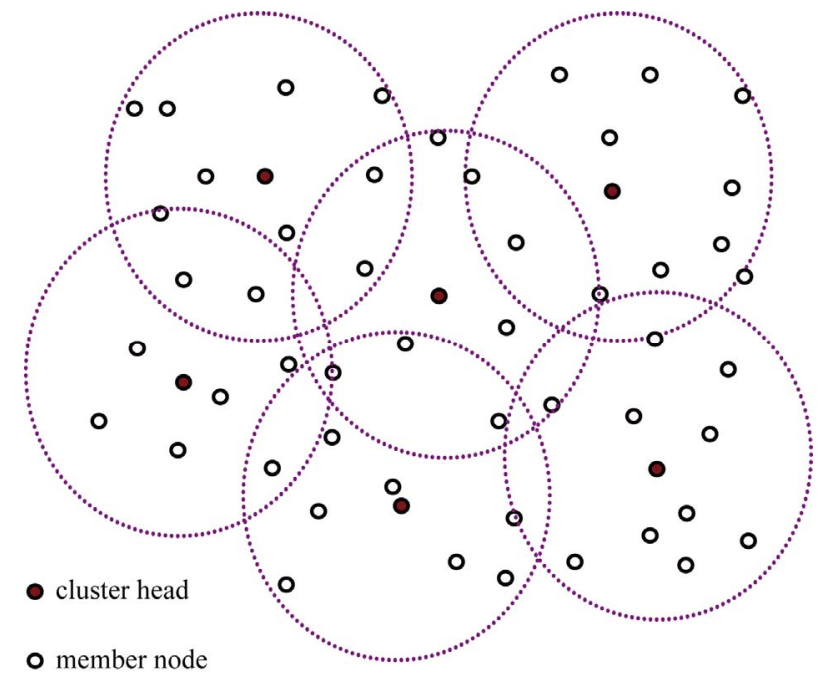

Figure 1. An example of the network model.

targets localization.

\subsection{Data Collection}

Sensor nodes in the network are organized as a hierarchy of clusters. The entire network is divided into multiple clusters, and there are one cluster head node and multiple member nodes in each cluster. Each cluster head keeps a list of its member nodes. As soon as a target appears in a local area, all nodes receive the signal emitted by the target generate sensed data, then transmit it to their cluster head nodes, respectively.

When a cluster head receives the packet from a member node, it fires a waiting timer $T_{w}$. Before reporting sensed data to the base-station, it waits for $T_{w}$ time to collect packets from member nodes that have sent messages to it. This timer scheme can release packets lost resulted by data collision in a certain degree. A larger $T_{w}$ would allow larger latency in collecting sensed data and obtaining tracking results for the base-station. On the other hand, a larger $T_{w}$ gives the cluster heads more chances to collect enough data to locate targets with certain precision. Thus, the trade-off exists between tracking latency and precision. We thus allow applications to set an upper bound for this delay. In other words, applications can choose the trade-off adaptively.

After $T_{w}$ time, cluster heads begin to process data received from member nodes.

\subsection{Data Compressing}

We assumed that a cluster head has $m$ members, which have been sorted as node id ascending in member list stored over the cluster head and base-station, respectively. The data received from member nodes is represented 
as $N X=\left\{\left(n_{1}, X_{1}\right), \cdots,\left(n_{j}, X_{j}\right), \cdots,\left(n_{m}, X_{m}\right)\right\}$, where $n_{j}$ is the node $i d$ and $j$ is the serial number of node $n_{j}$ in member list, $j \in[1, m] . X_{j}$ is a $d$-dimensional vector $\left(x_{1 j}, \cdots, x_{k j}, \cdots, x_{d j}\right)$, where $d$ is the number of targets in the monitoring area and $x_{k j}$ is the reading of target $k$, $k \in[1, d]$. If the cluster head does not receive data of target $k$ from node $n_{j}$, it sets $x_{k j}-1$.

The cluster head compresses data from its member nodes as a compact structure with three entries:

- mean: it defines the mean value of data from all member nodes.

- bitmap: it is a map indicating if the sensed data of a sensor node can be approximated to mean within a given compressing error bound $\varepsilon$. The $i$ th bit ( $i=$ $1,2, \cdots)$ is used to indicate if the data from $i$ th node can be approximated by mean. If it is, set the value of bit $i 1$, else keep it as 0 . For example, a cluster has four member nodes and their sensed data is 30 , $32,30,34$. Their expected value (mean) is 31.5 . If the given compressing error is 2, then bitmap is 1 (0001 in binary). As the first three data falls with $\varepsilon$ around mean, they can be replaced by mean, and the last value falls outside $\varepsilon$ of mean, the 4th bit of bitmap is updated to 1 .

- variance: it is an active array to store the variance of data when it falls more than the specified error constraint $\varepsilon$ away from mean.

After time $T_{w}$, the cluster head initializes bitmap as 0 . To simplify the presentation, we use $b_{1} b_{2} \cdots b_{m}$ to represent the bits of bitmap, where $b \mathbf{i} \in\{0,1\}$, it is the value of ith bit of bitmap.

Since the compressing mechanism is applied independently for each target, we only consider the sensed data of target $k$ for simplicity. First, the expected value of all data in $N X$ is computed, and then the cluster head validates whether the data in $N X$ can be replaced by mean with guarantee of compressing error. For each data $x_{j}^{k}$, if it is -1 , it means that the data of node $n_{j}$ is not received, then set $b_{i} 1$ and write 0 to variance. Otherwise, its variance of mean $\sigma_{k j}^{2}$ is calculated. If $\sigma_{k j}^{2} \leq \varepsilon$, the cluster head replaces $x_{j}^{k}$ by mean. Otherwise, set $b_{i} 1$ and write $\sigma_{k j}$ to variance sequentially. We observe that the number of 1 in bitmap shows the number the values have been written to variance.

Algorithm 1 describes the compressing algorithm. The cluster head computes the expected value of all received data for the same target and assigns it to mean. For each data $x_{k j}$, if it is within $\varepsilon$ of mean, then it can be filtered out. Otherwise, the jth bit of bitmap is set 1 , meanwhile the variance of $x_{j}^{k}$ and $\varepsilon$ is written to $v a$ riance [getIndex $(j)$ ]. getIndex () is a function returns the corresponding subscript of $\sigma_{k j}$ in variance by counting the number of 1 in $b_{1} \cdots b_{m}$.

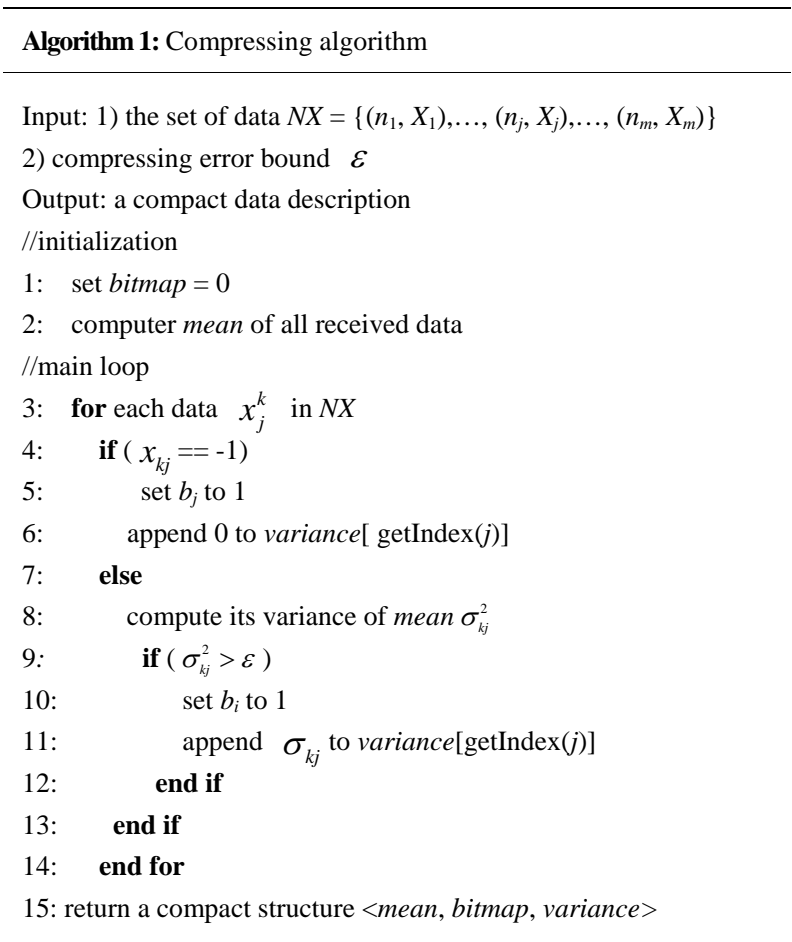

Now, we analyze the complexity of Algorithm 1. As the time complexity of function getIndex() is decided by the order of $x_{j}^{k}$ in $N X$. For the best case, it runs in $O(1)$ time, while for the worst case, it runs in $O(m)$, thus the average time complexity of function getIndex () is $O(m / 2)$. In Algorithm 1, computing the expected value of all received data requires $O(\mathrm{~m})$ time, and sentences 3 to 14 run in $O(m / 2+m \times m / 2)$ time, so the time complexity of the algorithm is $O\left(\mathrm{~m}^{2} / 4\right)$.

\subsection{Data Transmission}

After processing all packets from member nodes, the cluster head obtains a series of compact representation of sensed data for each target, which are transmitted to the base station.

\subsection{Targets Localization}

When the base-station has received the compressed data from a cluster head, it begins to locate targets. Most existing localizations algorithms can be incorporated with our protocol. Without generality, we adopt Centroid localization algorithm, which is attractive for its simplicity. Centroid localization algorithm computes the average location of all sensor nodes detecting the target as the location of the target. While its quality may be not good enough as it assigns equal weight to each node without considering its distance to the target. Instead of treating all nodes equally, we compute the weighted average of 
participant nodes' locations. Sensor nodes are weighted under its distance from the target. Thus, a sensor close to the target will be assigned a higher value.

Upon receiving a compact data description $G_{k}$, target localization is implemented to locate target $k$. As the base-station keeps the member list of each cluster, we can scan $G_{k}$.bitmap to identify if a node in the list has contributed sensed data of target $k$, moreover if its value has been replaced by $G_{k}$.mean.

Algorithm 2 presents the algorithm of targets localization on $G_{k}$, the algorithm is implemented on the compressed data directly. $F($.$) is a function converting the$ signal strength a sensor samples into the distance between the sensor and moving target emitting signal. We use $\frac{1}{F\left(x_{i}\right)^{d}}$ to denote the weight of node $n_{i}$, where $x_{i}$ is the sensed data generated by $n_{i}$, exponent $d$ is typically set as 1. Algorithm 2 runs in $O(m)$ time.

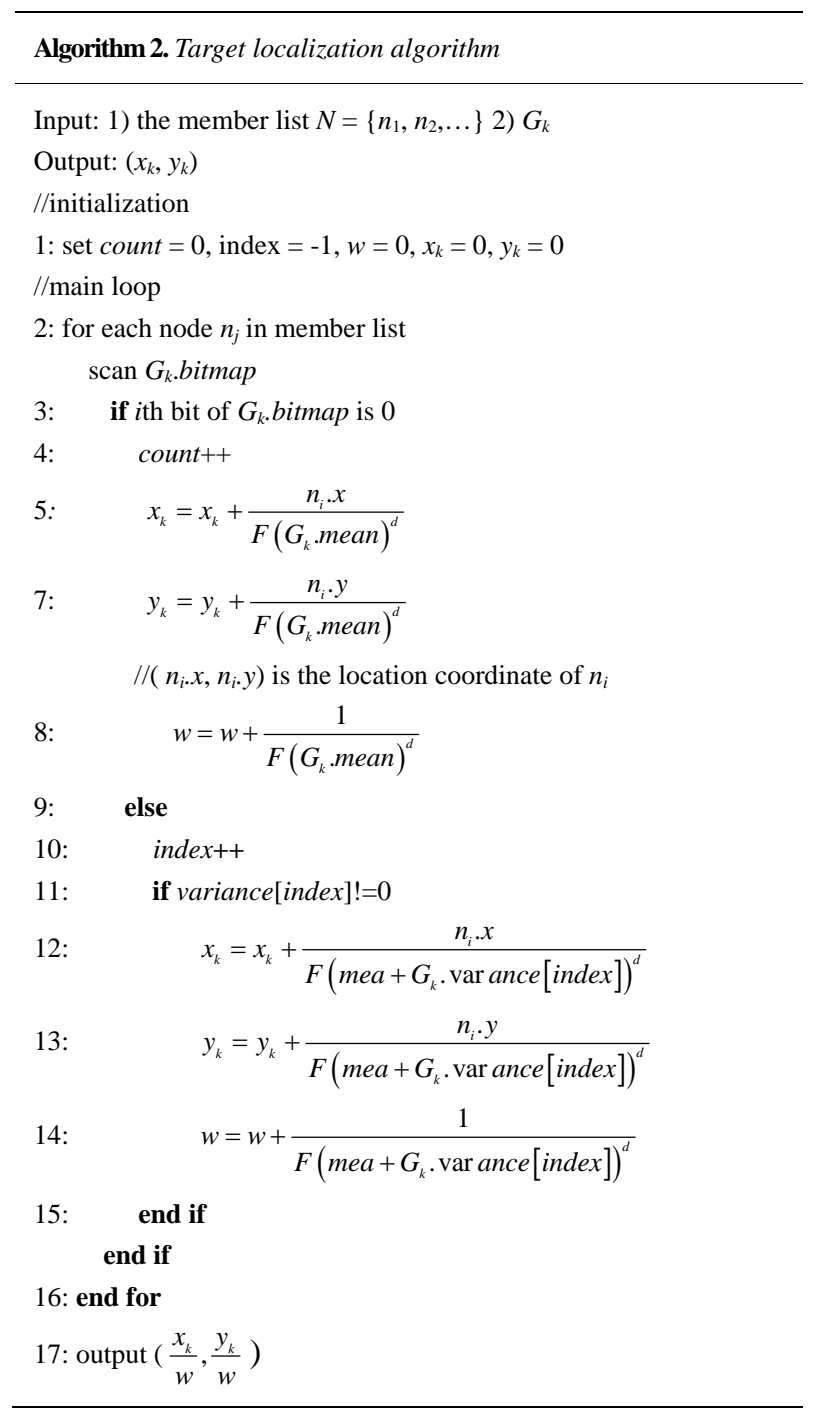

\section{Analysis of DCTTP}

In this section, we analyze the characteristic of DCTTP, and further discuss the trade-off between tracking quality and energy conservation.

We first define two metrics to measure the performance of DCTTP.

Definition 1 (Compressing error): Compressing error is the error between the real sample and its estimated value.

Definition 2 (Compression ratio): Compression ratio is the size of compact data description over the size of original data.

Let $\rho$ denote the density of the network. As sensor nodes are uniformly and independently distributed in the sensing area, the number of sensor nodes located in any subarea $s$, denoted as $N(s)$, follows Possion distribution with mean of $\rho\|s\|$, where

$$
P(N(s)=i)=e^{-\rho\|s\| \frac{(\rho\|s\|)^{i}}{i !}}
$$

When a moving target shows up in a local area, only nodes of which sensing disk cover the target generate sensed data. These nodes locate in the disk centered at the location of the moving target with radius $R$, then the number of nodes that can detect the target can be represented as:

$$
P(N(s)=i)=e^{-\rho \pi R^{2} \frac{\left(\rho \pi R^{2}\right)^{i}}{i !}}
$$

Thus, the number of nodes that can detect the target is $\sum P(N(s)=i)=\rho \pi R^{2}$.

Definition 3 (Detecting area): Detecting area is a local area, nodes in which can detect the target. It is a disk centered at the target with radius $R$.

Definition 4 (Dividing disk) Dividing disk is a disk centered at the target with radius $r$, denoted as $D D_{r}$. shortly.

Definition 5 (Detecting cirque) Detecting cirque is a subarea formed by dividing disk $D D_{(i+1) \varepsilon}$ excluding dividing disk $D D_{i \varepsilon}$, denoted as $D C_{i \rightarrow(i+1)}$.

According to the design of DCTTP, only the sensed data that fluctuates over their expected value can be replaced by mean. We divide the detecting area into a subset of areas by a serial of dividing disks with radius $\varepsilon, 2 \varepsilon, \cdots,\left\lceil\frac{R}{\varepsilon}\right\rceil \varepsilon$, respectively, as shown in Figure 2 . Nodes in the same subarea trend to have similar sensed data, that's most of them fluctuate over their expected values, compressing these data together can obtain better compressing ratio.

In the area of $D C_{i \rightarrow(i+1)}$, there are $\rho \pi(2 i \varepsilon+1) R^{2}$ 


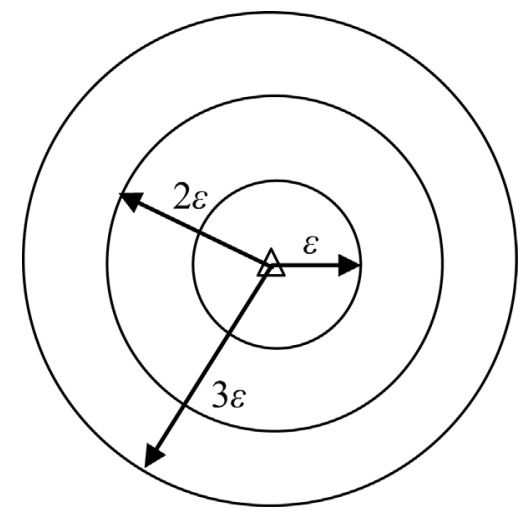

Figure 2. The figure of the sensing area division.

member nodes. Assume that $a$ units are needed to represent the sensed data and node $i d$, thus, a cluster head node need transmit $2 a \rho \pi(2 i \varepsilon+1) R^{2}$ units data to report the readings of one target. While with DCTTP, the cluster head transmits a compact data description of $a+\rho \pi(2 i \varepsilon+1) R^{2}+(1-p) \rho \pi(2 i \varepsilon+1) R^{2} a$ units, where $p$ is the percentage of the filtered data and $(1-p) \rho \pi(2 i \varepsilon+1) R^{2}$ is the number of values written to variance. $a$ units are used to represent mean and $\rho \pi(2 i \varepsilon+1) R^{2}$ units are used to represent bitmap, respectively.

The compression ratio of data in $D C_{i \rightarrow(i+1)}$, denoted as $C R_{i \rightarrow(i+1)}$ can be defined as:

$$
C R_{i \rightarrow(i+1)}=\frac{a+\rho \pi(2 i \varepsilon+1) R^{2}+(1-p) \rho \pi(2 i \varepsilon+1) R^{2} a}{2 a \rho \pi(2 i \varepsilon+1) R^{2}}
$$

Setting $a=64$, then we have:

$$
C R_{i \rightarrow(i+1)}=\frac{1}{64}+\frac{1-p}{2}+\frac{16}{\rho \pi(i \varepsilon+1) R^{2}}
$$

Thus, the compression ratio of data in the whole detecting area is:

$$
\begin{aligned}
C R_{\text {whole }} & =\sum_{i=1}^{\left\lceil\frac{R}{\varepsilon}\right\rceil}\left(\frac{1}{64}+\frac{1-p}{2}+\frac{16}{\rho \pi(i \varepsilon+1) R^{2}}\right) \\
& =\frac{33-32 p}{64}\left\lceil\frac{R}{\varepsilon}\right\rceil+\frac{16 \ln \left\lceil\frac{R}{\varepsilon}\right\rceil}{\rho \pi(\varepsilon+1) R^{2}}
\end{aligned}
$$

In formula (5), parameters $R$ and $p$ are fixed when the network is being deployed. It is clearly that $\varepsilon$ and $p$ are two key factors decide the compressing ratio, further energy conservation of the network.

In theory, sensed data of nodes in the same detecting cirque can be replaced by their expected value with va- riances less than $\varepsilon$ However, measurement errors and data noisy make variances of parts data beyond $\varepsilon$, these data has to be stored into the item variance of the compact structures.

As the measurement error and data noisy at a certain node usually follow Gaussian distribution [17]. As $p$ is decided by the distribution of data and $\varepsilon$, thus compressing ratio is in inverse proportion to the given compressing error bound $\varepsilon$. That's energy conservation is at the cost of data quality sacrificing. We can choose appropriate compressing error according to the moving mode of targets and experiences to obtain optimal performance of the system.

\section{Experiments and Evaluation}

In this section, we report our simulation results under two scenarios: with data noisy and without noisy. In each case, we report the performance of tracking quality and energy conservation and our analysis.

In the simulations, sensors nodes are deployed in a region of $1000 \times 1000$ unit field. The locations of nodes are known. All sensor nodes have the same sensing radius. Three electronic cars are simulated as the targets, which move along any velocity. For the case with noisy, we set the mean and variance of Gaussian noise at each sensor node to 1 .

We first define two metrics to measure the performance of DCTTP in terms of tracking quality and energy conservation, that's tracking error and energy savings ratio.

Tracking error: It is the average distance between the real trace of the moving target and its estimated trace.

Energy savings ratio: It is defined as the ratio of energy savings of DCTTP over the normal tracking system without energy conservation mechanisms.

In each scenario, we explore the impact of some key system configurations on the system performance, such as network density, sensing range and compressing error.

\subsection{Tracking Error}

Figure 3 shows the results of tracking error for varying network density. Fixing sensing range and compressing error, the number of sensor nodes is ranging from 100 to 1000 , and the corresponding density varies from $10^{4}$ to $10^{3}$. As the increasing of network density, tracking error decreases obviously. This is because when the network density is higher, there are more sensor nodes participating in locating the targets, which generates more sensed data to be involved in locating multiple targets. The influence of network density on data with noisy considering is more serious. As more sensed data helps to fix the 
error of data, which contributes to data that are more precise.

Figure 4 shows the influence of sensing range on tracking error. We observe that sensing range is one of key factors that influence tracking quality. As the raising of sensing range, more sensor nodes can detect the targets, which provides better tracking performance. It is observed that tracking error decrease slightly as the increase of compressing error, especially for data with noisy. From the results, we can conclude that: 1) compressing data does not bring obvious tracking error; 2) our compressing technique can efficient alleviate the impact of data noisy on tracking quality.

Figure 5 presents the influence of compressing ratio on tracking error. It is clear that tracking error is in inverse proportion to compressing ratio, while when compressing ratio reaches $50 \%$, the change of tracking error approaches to constant.

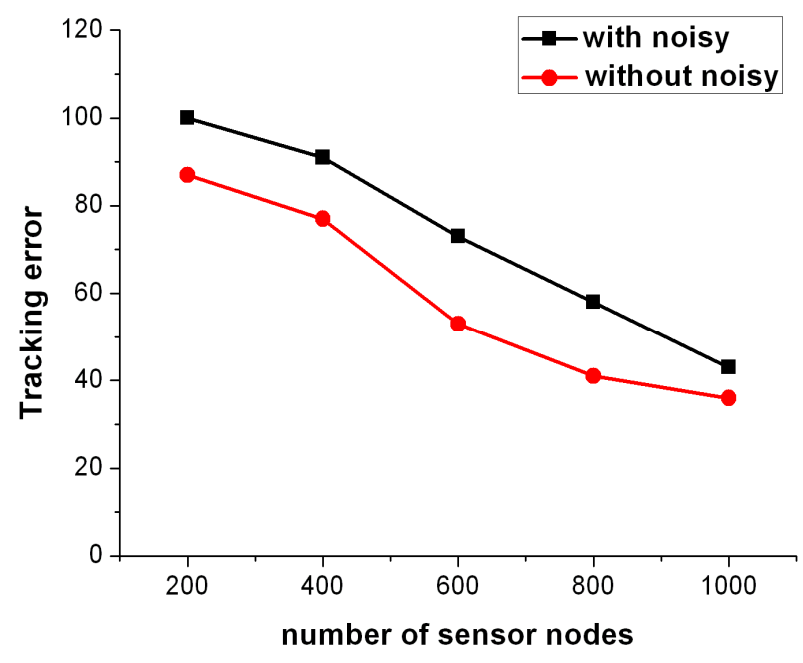

Figure 3. Impact of network density on tracking error.

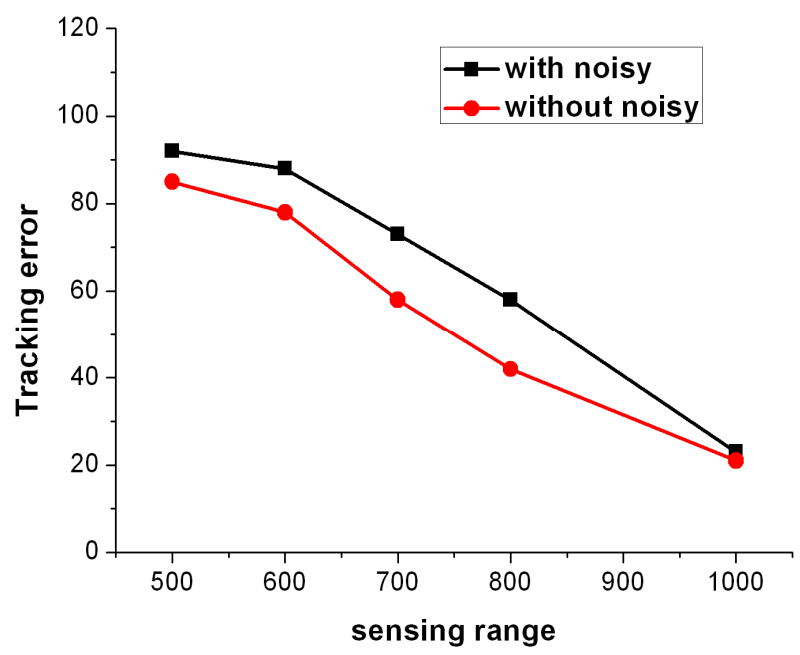

Figure 4. Impact of sensing range on tracking error.

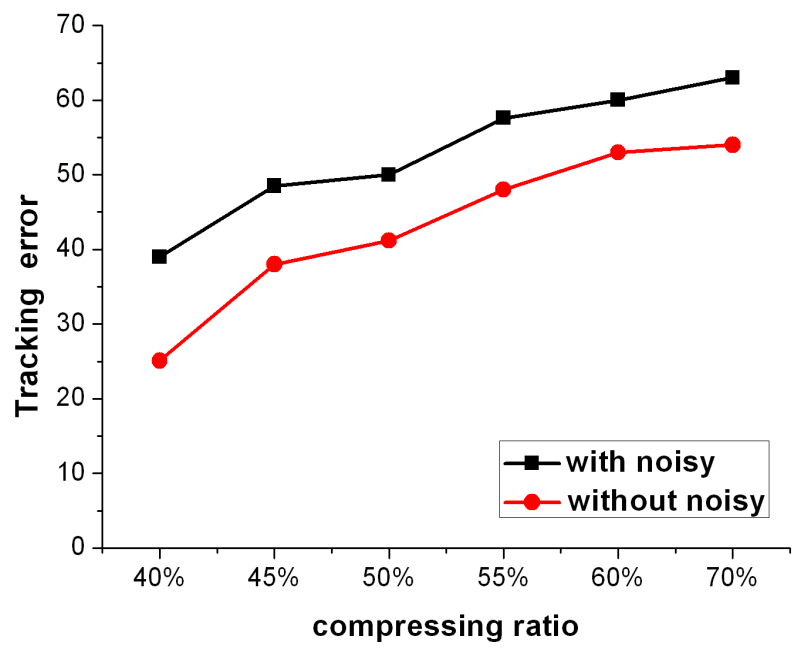

Figure 5. Impact of compressing ratio on tracking error.

\subsection{Energy Savings Ratio}

Figure 6 depicts the impact of network density on energy savings. Clearly, applying data compressing algorithm conserves much energy. As the increase of network density, energy savings enhances significantly. For increasing network density leads to more sensed data to be compressed, more energy of packets transmission is saved.

From Figure 7, we observe that energy saving increases monotonically with the raise of sensing range. The reason is that the increase of sensing range leads to more sensor nodes detecting the target, and more information transmitted to the base station, thus, the advantage of data compressing is more remarkable.

Figure 8 plots the influence of compressing ratio on energy savings. It is shown that the degree of data compressing also has influences on energy savings, especially

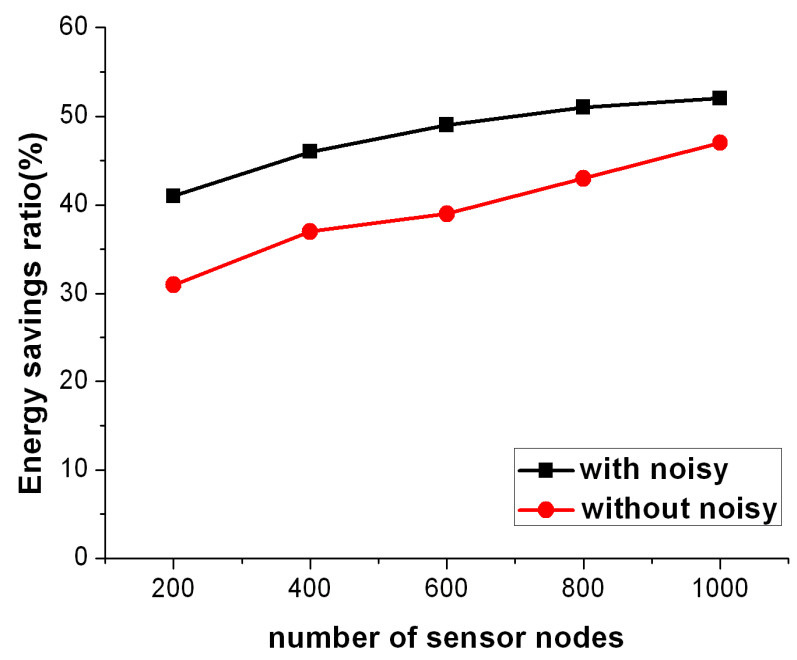

Figure 6. Impact of network density on energy saving ratio. 


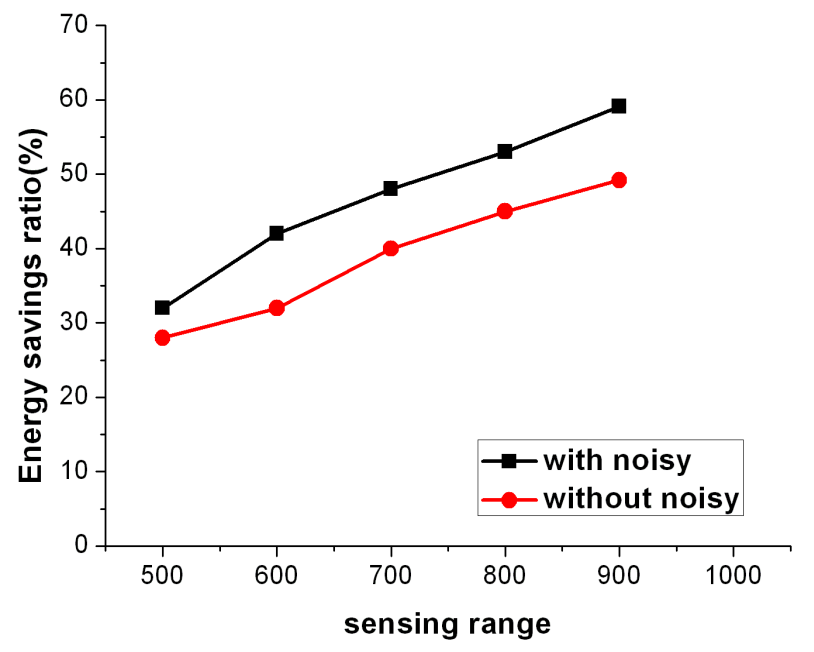

Figure 7. Impact of sensing range on energy savings ratio.

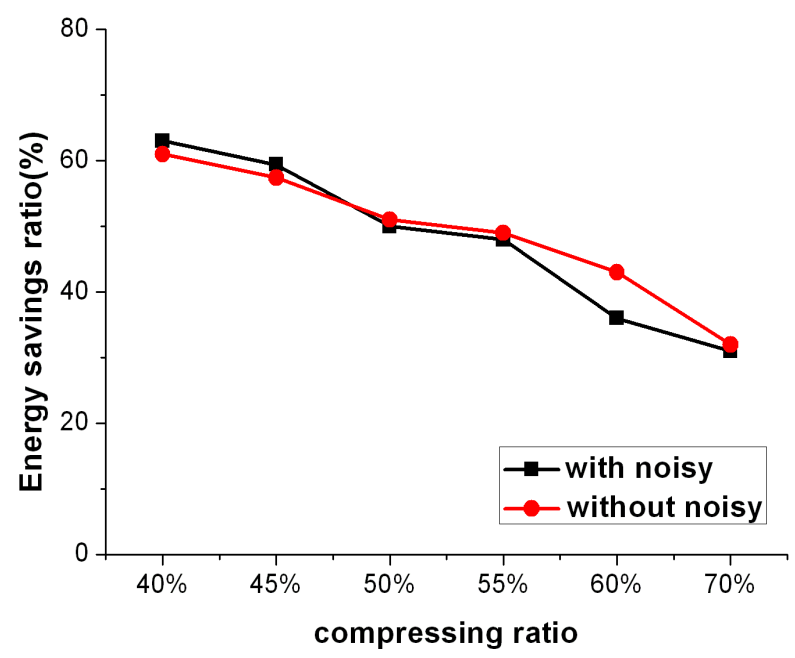

Figure 8. Impact of compressing ratio on energy savings ratio.

for data with noisy.

\section{Conclusions}

In this paper, we concentrate on energy efficient tracking, dedicated to conserve the whole network energy, as well as maintain high tracking quality. We have proposed a data compressing scheme to reduce information transmission of targets. In addition, we incorporate the proposed data compressing technique with tracking protocol and optimize it to obtain trade-off between energy conservation and tracking quality.

We implement a set of simulations to validate our approach. The results demonstrate the effectiveness of proposed protocol and illustrate influences of several parameters on the system. As our future work, we will implement our tracking algorithm on real sensor nodes.

\section{Acknowledgements}

This work is partially supported by the NSFC-RGC of China under Grant No.60831160525, the Key Program of the National Natural Science Foundation of China under Grant No. 61033015, the National Natural Science Foundation of China under Grant No. 60933001, the National Natural Science Foundation of China under Grant No. 60703012, the Natural Science Foundation of Heilongjiang Province of China under Grant No. F201038 and the Science and Technology Innovation Research Project of Harbin for Young Scholar under Grant No. 2009RFQX080.

\section{References}

[1] Q. Ren, J. Li and H. Gao, “Tpss: A Two-Phase Sleep Scheduling Protocol for Object Tracking in Sensor Networks," Proceedings of 6th IEEE International Conference on Mobile Ad-hoc and Sensor Systems, Macao, 2009, pp. 458-465.

[2] Z. Zhong, T. Zhu, D. Wang and T. He, "Tracking with Unreliable Node Sequences,” INFOCOM, Rio de Janeiro, 19-25 April 2009, pp.1215-1223 .

[3] J. Jeong, T. Hwang, T. He and D. Du, "MCTA: Target Tracking Algorithm Based on Minimal Contour in Wireless Sensor Networks,” Proceedings of 26th Conference on Computer Communications, Anchorage, 6-12 May 2007, pp. 2371-2375.

[4] T. He, P. A. Vicaire, T. Yan, L. Luo, L. Gu, G. Zhou, R. Stoleru, Q. Cao, J. A. Stankovic and T. Abdelzaher, "Achieving Real-Time Target Tracking Using Wireless Sensor Networks," ACM Transaction on Embedded Computing System, 2007.

[5] T. He, P. Vicaire, T. Yan, Q. Cao, G. Zhou, L. Gu, L. Luo, R. Stoleru, J. A. Stankovic and T. Abdelzaher, “Achieving Long-Term Surveillance in VigilNet," Proceedings of 25th Conference on Computer Communications, Barcelona, April 2006, pp. 1-12.

[6] N. Shrivastava, R. Mudumbai, U. Madhow and S. Suri, "Target Tracking with Binary Proximity Sensors: Fundamental Limits, Minimal Description, and Algorith," ACM Sensys, November 2006.

[7] W. Kim, K. Mechitov, J.-Y. Choi and S. K. Ham, "On Tracking Objects with Binary Proximity Sensors,” Proceedings of 4th International Conference on Information Processing in Sensor Networks, April 2005.

[8] J. Singh, U. Madhow, R. Kumar, S. Suri and R. Cagley, "Tracking Multiple Targets Using Binary Proximity Sensors," Proceedings of 6th International Conference on Information Processing in Sensor Networks, April 2007. doi:10.1145/1236360.1236427

[9] Z. J. Wang, E. Bulut, B. K. Szymansky, "Distributed Energy-Efficient Target Tracking with Binary Sensor Networks," ACM Transactions on Sensor Networks, Vol. 6, No. 4, July 2010. doi:10.1145/1777406.1777411 
[10] W. S. Zhang and G. H. Cao, "DCTC: Dynamic Convey Tree-based Collaboration for Target Tracking in Sensor Networks," IEEE Transactions on Wireless Communication, Vol. 3, No. 5, September 2004, pp. 1689-1701. doi: 10.1109/TWC.2004.833443

[11] F. Zhao, J. Shin and J. Reich, "Information-driven Dynamic Sensor Collaboration for Tracking Applications,” IEEE Signal Processing Magazine, Vol. 19, No. 2, March 2002, pp. 61-72. doi:10.1109/79.985685

[12] B. Kusy, A. Ledeczi and X. Koutsoukos, “Tracking Mobile Nodes Using RF Doppler Shifts,” Proceedings of 5th International Conference on Embedded Networked Sensor Systems, 2007, pp. 29-42. doi:10.1145/1322263.1322 267

[13] R. Sarkar and J. Gao, "Differential Forms for Target Tracking and Aggregate Queries in Distributed Networks," Proceedings of 11th ACM International Symposium on Mobile Ad hoc Networking and Computing, 2010, pp. 377-388.

[14] Q. Cao, T. Abdelzaher, T. He and J. Stankovic, “Towards
Optimal Sleep Scheduling in Sensor Networks for Rare-Event Detection," Proceedings of the 4th International Symposium on Information Processing in Sensor Networks, 15 April 2005, pp. 20-27.

[15] C. Gui and P. Mohapatra, "Power conservation and Quality of Surveillance in Target Tracking Sensor Networks," Proceedings of the International Conference on Mobile Computing and Networking, 2004.

[16] W. Heinzelman, A. Chandrakasan and H. Balakrishnan, "Energy-efficient Communication Protocol for Wireless Micro-sensor Networks," Proceedings of the 33rd International Conference on System Sciences, Vol. 8, January 2000.

[17] G. Xing, R. Tan, B. Liu, J. Wang, X. Jia and C. W. Yi, "Data Fusion Improves the Coverage of Wireless Sensor Networks," Proceedings of 15th Annual International Conference on Mobile Computing and Networking, New York, 2009, pp. 157-168. doi:10.1145/1614320.161 4338 